MODELING, IDENTIFICATION AND CONTROL, 1988, vOL. 9, NO. 2, 81-97

doi:10.4173/mic.1988.23

\title{
Maximum likelihood estimation of ocean wave spectra from general arrays of wave gauges
}

\author{
HARALD E. KROGSTAD $\dagger$ \\ Keywords: Maximum Likelihood Method (MLM), wave spectra, arrays

\begin{abstract}
The paper discusses estimation of directional ocean wave spectra from a spatial array of sensors by means of the Maximum Likelihood (ML) method. An iterative improvement of the ML estimate is proposed and illustrated on synthetic and real data. The wave data was obtained by an acoustic Doppler current meter in the Current Measurement Experiment (CUMEX) carried out by Esso Norge a.s at the Odin platform in the Norwegian Sea.
\end{abstract}

\section{Introduction}

The wind generated gravity waves on the ocean surface are of major concern in naval architecture and ocean engineering. However, since the complexity of wave motion is evident with wave patterns that are constantly changing in time and space, any mathematical model has to be of a stochastic nature. Given appropriate temporal and spatial scales, the surface may be considered to be a stationary and homogeneous Gaussian stochastic field. The wavenumber-frequency spectrum of the field thereby becomes the basic quantity which enters computations of the response of offshore structures and ship motion.

Wave measurements have traditionally been limited to single point measurements of the surface elevation by means of buoys or wave staffs. Today, however, multichannel measurements which reveal directional properties of the spectrum are becoming routine and heave/pitch/roll buoys have proved to be robust and reliable instruments for long term data acquisition (Barstow and Krogstad, 1984). On fixed structures, vector measuring current meters, often combined with pressure cells or wave staffs are commonly used (Borgman, 1982a). Arrays of wave gauges have so far mainly been used for research purposes, but new concepts, in particular small arrays of infrared laser altimeters and the acoustic doppler current profiler may soon change this state of affairs.

The present paper summarizes some of the methods that are used to process data from arrays of wave gauges, mainly focusing on the Maximum Likelihood Method (MLM) and an iterative refinement to it. The methods are illustrated using data from the CUrrent Measurement EXperiment (CUMEX). CUMEX is a joint programme between Esso Norge a.s and Exxon Production Research Company with the objective of instrumenting Esso's Odin platform in the Norwegian Sea to study the characteristics of ocean currents generated by severe storms. The instrumentation includes four Acoustic Doppler Current Meters (ADCM) developed by R\&D Instruments, San Diego (Gordon and Skorstad, 1985). These ADCMs are

Received 10 September 1987.

† OCEANOR, Oceanographic Company of Norway, P.O. Box 2905 - Tempe, N-7002 Trondheim, Norway. 
designed to record the along-beam velocity at 25 equally spaced positions along two horizontal orthogonal beams. Since the wavelength of the shortest observable water waves is shown to be $35 \mathrm{~m}$ as opposed to the array spacing of $4.6 \mathrm{~m}$, there is a large amount of redundancy in the data sets which requires numerical modifications to the algorithms that had been used previously. For a more complete description of the measurement system and the iterative MLM applied to a larger set of data from CUMEX, we refer to Krogstad, Miller and Gordon, (1988).

\section{The stochastic model}

Consider a Cartesian coordinate system with horizontal $x$ and $y$ coordinate axes, the $z$-axis pointing upwards, and the origin at the mean water level. The ocean surface is denoted $z=\eta(x, t), x=(x, y), t=$ time. With appropriate temporal and spatial scales the surface is a zero mean, second order homogeneous and stationary stochastic field with a covariance function $\rho(x, t)$. The spectrum of the field, $\chi(k, \omega)$, is a function of wavenumber $\boldsymbol{k}$ and frequency $\omega$ :

$$
\rho(x, t)=\int_{\boldsymbol{k}} \int_{\omega} \exp (\mathrm{i}(\boldsymbol{k} \cdot \boldsymbol{x}-\omega t)) d \chi(\boldsymbol{k}, \omega)
$$

We recall the spectral representation of the field:

$$
\eta(x, t)=\int_{\boldsymbol{k}} \int_{\omega} \exp (\mathrm{i}(\boldsymbol{k} \cdot \boldsymbol{x}-\omega t)) d B(\boldsymbol{k}, \omega)
$$

where $B(k, \omega)$ is the spectral amplitude (Gihman and Skorohod, 1974).

The term 'wave spectrum' is commonly used for the frequency spectrum, $S_{\eta}(\omega)$, defined by

$$
\rho_{\eta}(0, t)=\int_{-\infty}^{+\infty} \exp (-\mathrm{i} \omega t) S_{\eta}(\omega) d \omega
$$

Hence, formally,

$$
S_{\eta}(\omega) d \omega=\int_{\boldsymbol{k}} d \chi(\boldsymbol{k}, \omega) .
$$

Measurements have revealed that gravity waves on the ocean surface are very close to a Gaussian field. In fact, this is probably among the phenomena in nature closest to the realization of a Gaussian field, see Fig. 1.

If we assume incompressible, inviscid and irrotational conditions, the surface, $\eta$, and the velocity potential, $\Phi(u(x, z, t)=\nabla \Phi)$, satisfy to the first order in characteristic wave steepness (Philips, 1977)

$$
\left.\begin{array}{rl}
\omega^{2} & =g k \tanh (k h), k=|k|, h=\text { mean water depth } \\
\eta(x, t) & =\int_{k} \int_{\omega} \exp (\mathrm{i}(\boldsymbol{k} \cdot \boldsymbol{x}-\omega t)) d B(k, \omega) \\
\Phi(x, z, t) & =\int_{k} \int_{\omega} \frac{-\mathrm{i} \omega}{k} \frac{\cosh (k(z+h))}{\sinh (k h)} \exp (\mathrm{i}(\boldsymbol{k} \cdot \boldsymbol{x}-\omega t)) d B(k, \omega)
\end{array}\right\}
$$

This is usually referred to as the linear stochastic model. Experience has shown that the linear stochastic model is a good approximation for the dominant parts of the field. 


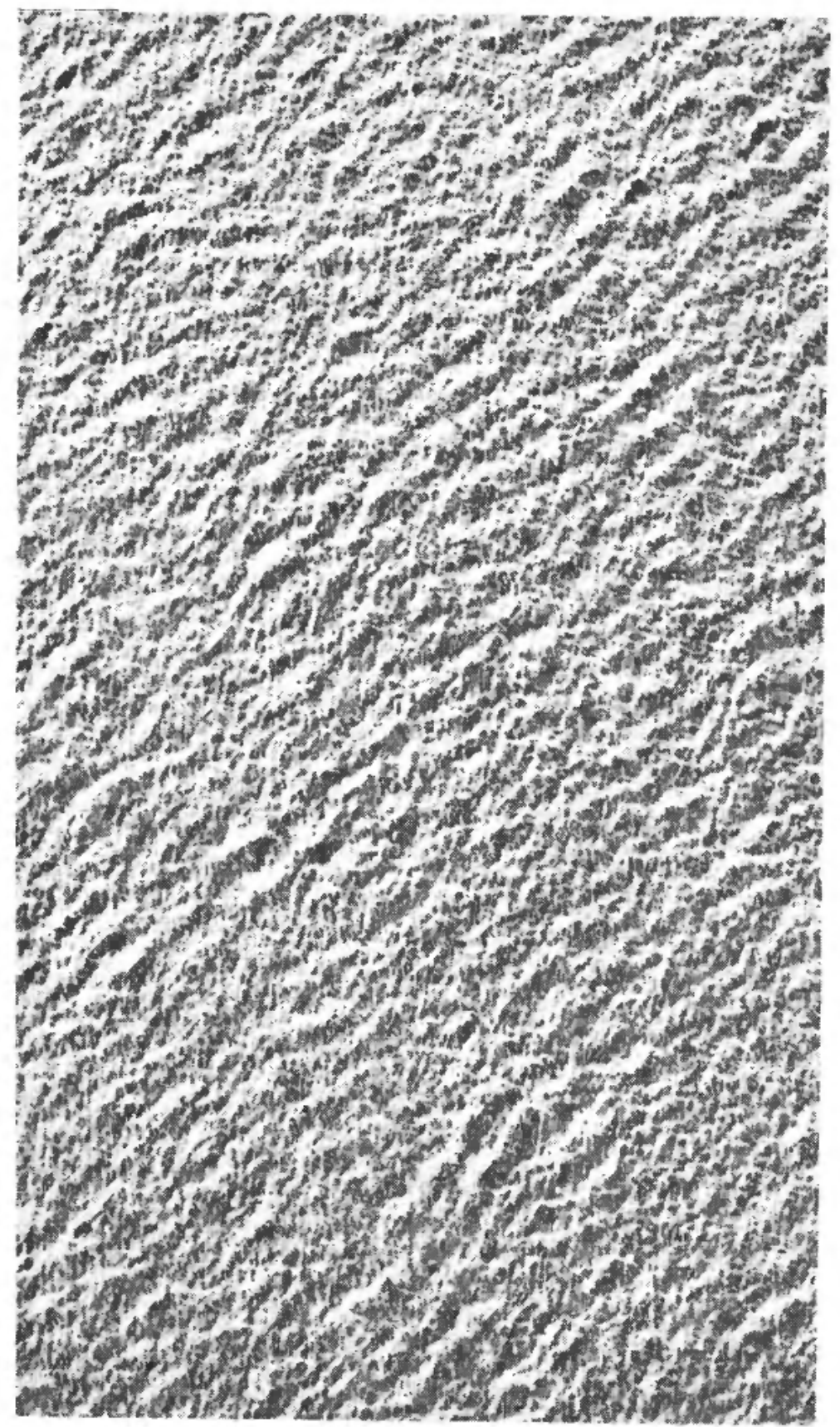

Figure 1. Aerial imagery of ocean waves illustrating the randomness of the sea (Courtesy Norsk luftfoto og fjernmåling $\mathrm{A} / \mathrm{S}$, Oslo)

The spectrum in the linear model is supported by the surface $\omega^{2}=g k \cdot \tanh (k h)$ and may be expressed in terms of $\omega$ and the direction, $\theta$, of $k$, formally,

$$
d \chi(\boldsymbol{k}, \omega)=S(\omega) D(\theta, \omega) d \omega d \theta
$$

Analytical expressions for $S$ are discussed in Kinsman (1965) and Phillips (1977). Borgman (1979) and Barstow and Krogstad (1984) list some suggested analytical 
expressions for $D$. In practice, with several co-existing wave fields, neither $S$ nor $D$ have simple shapes.

In the linear stochastic model, quantities such as surface elevation, surface slope, wave induced water velocity, dynamic pressure at a certain location are related to $\eta(x, t)$ by linear filters mapping the surface into a corresponding stochastic process, $X$,

$$
X(t)=\int_{k} \int_{\omega} T_{X}(k, z, \omega) \exp \left(\mathrm{i}\left(k \cdot x_{0}-\omega t\right)\right) d B(k, \omega)
$$

where $T_{X} \exp \left(\mathrm{i} k \cdot x_{0}\right)$ is the transfer function for the quantity in question. Apart from the factor $\exp \left(\mathrm{i} k \cdot x_{0}\right)$, the following transfer functions are easily derived (Borgman, 1979)

Surface elevation: $T_{\eta}=1$

Slope in $x$-direction: $T_{\eta_{\mathrm{x}}}=\mathrm{i} k \cos \theta$

Slope in $y$-direction: $T_{\eta_{y}}=\mathrm{i} k \sin \theta$

Velocity in x-direction: $T_{u}=\omega \frac{\cosh (k(z+h))}{\sinh (k h)} \cos \theta$

Velocity in $y$-direction: $T_{v}=\omega \frac{\cosh (k(z+h))}{\sinh (k h)} \sin \theta$

Dynamic pressure $(p=-\rho \partial \Phi / \partial t): T_{p}=\rho \frac{\omega^{2}}{k} \frac{\cosh (k(z+h))}{\sinh (k h)}$

All transfer functions (now including $\exp \left(\mathrm{i} k \cdot x_{0}\right)$ ) can be written $T=R h$ where $R$ is independent of $\theta$ and $h$ may depend on $\omega$ through the factor $\exp \left(\mathrm{i} k \cdot x_{0}\right)$.

The cross-spectral matrix, $\Sigma=\Sigma(\omega)=\left\{\sigma_{i j}(\omega)\right\}_{i, j=1}^{N}$ for a set of quantities, $\left\{X_{i}\right\}_{i=1}^{N}$, is

$$
\Sigma(\omega)=\int_{\boldsymbol{k}} T(\boldsymbol{k}, \omega) T^{H}(\boldsymbol{k}, \omega) d \chi(\boldsymbol{k}, \omega)
$$

where $T=\left(T_{1}, T_{2}, \ldots, T_{N}\right)^{\prime}$ and ' $H$ ' means Hermitian transposed. Obviously,

$$
\sigma_{i j}(\omega)=S(\omega) R_{i}(\omega) R_{j}^{*}(\omega) \int_{0}^{2 \pi} h_{i}(\theta) h_{j}^{*}(\theta) D(\theta) d \theta
$$

where the $z$ - and $\omega$-dependences are suppressed when self-evident.

In the following, we focus on the estimation of the directional distribution. In practice, the recording of $\left\{X_{i}\right\}_{i=1}^{N}$ may involve filters of a hydrodynamic or electronic origin in addition to the basic wave theory filters.

\section{Estimation techniques}

\subsection{General considerations}

The starting point is a set of $N$ measured time series, $\left\{W_{i}\right\}_{i=1}^{N}$ of some of the wave-related quantities discussed in the previous section. We shall assume that the series are recorded with a certain sampling frequency, $\omega_{s}$, with $M$ samples in each series. 
In practice, the recording interval, $2 \pi M / \omega_{s}$, will typically be rather long compared to the correlation time of the series. Thus, the series are 'well-recorded', and the asymptotic relations for the discrete Fourier coefficients $\hat{W}=\left\{\hat{W}_{i}\right\}_{i=1}^{N}$ apply to a high degree of accuracy (Hannan (1970), Brillinger (1975)).

(i) For each $\omega_{\alpha}=\alpha \omega_{s} / M,|\alpha|<M / 2, \alpha \neq 0,\left\{\hat{W}_{i}\left(\omega_{\alpha}\right)\right\}_{i=1}^{N}$ are zero mean jointly complex Gaussian variables, with a covariance matrix defined by Eqn. (2.9).

(ii) $\hat{W}\left(\omega_{\alpha}\right)$ is independent of $\hat{W}\left(\omega_{\alpha^{\prime}}\right)$ if $\alpha \neq \pm \alpha^{\prime}$.

The standard estimate of the cross-spectral matrix is

$$
\hat{\Sigma}=\frac{2}{v} \sum_{\beta=1}^{v / 2} \hat{W}\left(\omega_{\beta}\right) \hat{W}^{H}\left(\omega_{\beta}\right)
$$

where $\omega_{\beta}$ runs over $\omega_{\alpha}$ s surrounding the frequency in question. $\hat{\Sigma}$ has asymptotically a complex Wishart distribution with $v$ degrees of freedom, and, moreover

(i) $E(\hat{\Sigma})=\Sigma$

(ii) $\operatorname{Cov}\left(\hat{\sigma}_{i j}\left(\omega_{\alpha}\right), \hat{\sigma}_{k m}\left(\omega_{\alpha}\right)\right)=\frac{2}{v} \sigma_{i k}\left(\omega_{\alpha}\right) \sigma_{j m}^{*}\left(\omega_{\alpha}\right), \omega_{\alpha} \neq 0, \omega_{s} / 2$

(iii) $\operatorname{Cov}\left(\hat{\sigma}_{i j}\left(\omega_{\alpha}\right), \hat{\sigma}_{k m}\left(\omega_{\alpha^{\prime}}\right)\right)=0$ for disjoint frequency bands.

From Equation (2.9) it follows that

$$
\Sigma=S R \Phi R^{H}
$$

where

$$
\begin{aligned}
& R=\operatorname{diag}\left(R_{1}, \ldots, R_{N}\right) \text { and } \\
& \Phi=\int_{0}^{2 \pi} h(\theta) h^{H}(\theta) D(\theta) d \theta .
\end{aligned}
$$

Given $\left\{\hat{W}\left(\omega_{1}\right), \ldots, \hat{W}\left(\omega_{v / 2}\right)\right\}$ as in Equation (3.1), the log likelihood function of $\Sigma, L(\hat{W}, \Sigma)$, is proportional to

$$
L(\hat{W}, \Sigma)=-\log |\Sigma|-\frac{2}{v} \sum_{\beta=1}^{v / 2} \hat{W}^{H}\left(\omega_{\beta}\right) \Sigma^{-1} \hat{W}\left(\omega_{\beta}\right)=-\log |\Sigma|-\operatorname{tr}\left(\Sigma^{-1} \hat{\Sigma}\right)
$$

and the maximum likelihood (ML) estimates of $S$ and $D$ are the solutions of

$$
L(\hat{W}, \Sigma)=\max
$$

subject to

$$
S \geqslant 0, D \geqslant 0, \int_{0}^{2 \pi} D(\theta) d \theta=1
$$

To our knowledge, no simple algorithm for a solution of (3.6) has appeared in oceanographic literature. The geometry of the array will generally impose certain constraints on the structure of $\Sigma$. $\Sigma$ is for instance a Toeplitz matrix for a uniformly spaced linear array of identical sensors. With no constraints on $\Sigma$, the ML estimate is simply $\hat{\boldsymbol{\Sigma}}$ (Mardia et al., 1979, pp. 102-108). 
For $\hat{\Sigma}$ as defined in (3.1), the equation for $\hat{D}$ and $\hat{S}$,

$$
\hat{\Sigma}=\hat{S} R\left(\int_{0}^{2 \pi} h(\theta) h^{H}(\theta) \hat{D}(\theta) d \theta\right) R^{H},
$$

does not necessarily have a solution. Even if we knew that $\hat{\Sigma}$ belonged to the class of 'admissible' matrices, the direct inversion of (3.7) is awkward.

\subsection{Linear and maximum likelihood estimates}

For the methods discussed below, multiplicative constants in the cross-spectral matrix are of no importance, and we shall work with estimates of $\Phi, \hat{\Phi}$, rather than $\Sigma$ (See Eqn. 3.3). Linear directional estimates have the form

$$
\hat{D}(\theta)=\gamma^{H}(\theta) \hat{\Phi} \gamma(\theta)
$$

where the weight factors $\gamma(\theta)$ are independent of $\Phi$ (Davis and Regier, 1977).

Since $\hat{\Phi}$ is positive semi-definite, $\hat{D}(\theta) \geqslant 0$ and

$$
E(\hat{D}(\theta))=\int_{0}^{2 \pi} K\left(\theta, \theta^{\prime}\right) D\left(\theta^{\prime}\right) d \theta^{\prime}
$$

where the integral kernel $K$ is

$$
\begin{aligned}
K\left(\theta, \theta^{\prime}\right) & =\gamma^{H}(\theta) h\left(\theta^{\prime}\right) h^{H}\left(\theta^{\prime}\right) \gamma(\theta) \\
& =\left|\gamma^{H}(\theta) h\left(\theta^{\prime}\right)\right|^{2}
\end{aligned}
$$

The objective is now to select weight factors $\gamma$ such that $K$ approximates a $\delta$ function situated at $\theta=\theta^{\prime}$. From Eqns (3.2) and (3.8), it follows easily that (Capon, 1969)

$$
\operatorname{Var}(\hat{D}(\theta)) \approx \frac{2}{v} E(\hat{D}(\theta))^{2}
$$

Thus, the variance of $\hat{D}(\theta)$ can only be reduced by increasing the averaging of the cross-spectra. Several optimizing algorithms for the weight factors are given in the literature (Davis and Regier, 1977, Capon, 1969, 1979). The following approach leads both to an optimal linear method and a high resolution method introduced by Capon (1969). Let the range of $\Phi$ as a linear map on $C_{N}$ be $R(\Phi)$ and $L=$ $\operatorname{span}_{D(\theta) \neq 0} h(\theta)$. We shall assume for simplicity that $h$ and $D$ are continuous. It is obvious that $R(\Phi) \subset L$ since $\Phi x=\int_{0}^{2 \pi} h(\theta)\left(h^{H}(\theta) x\right) D(\theta) d \theta \in c l(L)=L$. Conversely, if $x \in R(\Phi)^{\perp}$, then $0=x^{H} \Phi x=\int_{0}^{2 \pi}\left|h^{H}(\theta) x\right|^{2} D(\theta) d \theta$ and $h^{H}(\theta) x$ has to vanish whenever $D(\theta) \neq 0$, that is, $x \in L^{\perp}$. Hence $R(\Phi)=L$. Consider the following optimization problem:

$$
\gamma^{H} \Phi \gamma=\min \text { while }\left|\gamma^{H} h\right|^{2}=1
$$

Since $\exp (\mathrm{i} \alpha) \gamma_{s}, \alpha \in \boldsymbol{R}$, is a solution if $\gamma_{s}$ is a solution, the constraint may be replaced by $\gamma^{H} h=1$. The constraint thus imposes $\delta^{H} h=0$ for the variation $\delta$ around $\gamma_{s}$. Since

$$
\left(\gamma_{s}+\delta\right)^{H} \Phi\left(\gamma_{s}+\delta\right)=\gamma_{s}^{H} \Phi \gamma_{s}+2 \operatorname{Re}\left(\delta^{H} \Phi \gamma_{s}\right)+\delta^{H} \Phi \delta,
$$

$\gamma_{s}$ must be proportional to $\gamma_{0}$ where $\Phi \gamma_{0}=h$. Since $h \in R(\Phi)$, such a $\gamma_{0}$ always exists, in fact,

$$
\gamma_{0}=\Phi^{+} h+\gamma^{\perp}
$$


where $\Phi^{+}$is the Moore-Penrose inverse of $\Phi$ and $\gamma^{\perp} \in R(\Phi)^{\perp}$. The general solution (apart from a constant of modulus 1) of (3.12) may thus be written

$$
\gamma_{s}=\Phi^{g} h / h^{H} \Phi^{g} h
$$

where $\Phi^{g}$ is some generalized inverse of $\Phi$.

If we return to Eqn. (3.9) and the comment following Eqn. (3.10), an obvious optimizing criterion would be

$$
K(\theta, \theta)=\left|\gamma^{H}(\theta) h(\theta)\right|^{2}=\max
$$

while

$$
\begin{aligned}
\int_{0}^{2 \pi} K\left(\theta, \theta^{\prime}\right) d \theta^{\prime} & =\gamma^{H}(\theta) V \gamma(\theta)=1, \\
V & =\int_{0}^{2 \pi} h(\theta) h^{H}(\theta) d \theta .
\end{aligned}
$$

If $h(\theta) \neq 0$, the problem is equivalent to (3.12) with $D(\theta)=1 / 2 \pi$, and the solution is

$$
\gamma(\theta)=V^{g} h(\theta) /\left(\left(h^{H}(\theta) V^{g} h(\theta)\right)^{1 / 2}\right.
$$

leading to

$$
K\left(\theta, \theta^{\prime}\right)=\frac{\left|h^{H}(\theta) V^{g} h\left(\theta^{\prime}\right)\right|^{2}}{h^{H}(\theta) V^{g} h(\theta)}
$$

For the special case $h(\theta)=(1, \exp (\mathrm{i} \theta), \exp (2 \mathrm{i} \theta), \ldots, \exp (\mathrm{i} N \theta) t), K\left(\theta, \theta^{\prime}\right)=\sigma_{N}(\theta$ $-\theta^{\prime}$ ), the $N$ th Fejér kernel function.

Capon (1969) developed a high resolution method for seismic processing which later has become known as the maximum likelihood method (MLM) for directional spectra. The method was introduced in the context of ocean wave spectra by Davis and Regier (1977), Jeffreys et al. (1981), L. E. Borgman (1982) and Isobe et al. (1984). It is most easily explained by considering the optimal kernel for the case of one dominant, plane wave in direction $\theta$ superimposed on the background 'noise'. This amounts to

$$
K\left(\theta, \theta^{\prime}\right)=1
$$

while

$$
\int_{\theta^{\prime}=0}^{2 \pi} K\left(\theta, \theta^{\prime}\right) D\left(\theta^{\prime}\right) d \theta^{\prime}=\min ,
$$

the solution of which is again given by (3.14). The procedure is repeated for each direction, and the estimate, $D_{\mathrm{MLM}}$, is scaled to 1 afterwards:

$$
\begin{gathered}
D_{\mathrm{MLM}}(\theta)=\kappa \gamma^{H}(\theta) \Phi \gamma(\theta)=\kappa / h^{H}(\theta) \Phi^{g} h(\theta) \\
\kappa^{-1}=\int_{0}^{2 \pi}\left(h^{H}(\theta) \Phi^{g} h(\theta)\right)^{-1} d \theta
\end{gathered}
$$

In practice, an estimate $\hat{\Phi}$ for $\Phi$ is used, but (3.18) remains meaningful as long as $\hat{\Phi}$ is positive semi-definite. The method offers a choice for $\Phi^{g}$. L. E. Borgman (loc. cit.) obtained numerically stable estimates utilizing $\Phi^{+}$computed from the singular value decomposition of $\Phi$, whereas the present author has used Cholesky decomposition with diagonal pivoting followed by a reduction of the problem to channels 
that occur with non-zero pivots. This latter approach corresponds to renumbering the channels and using

$$
\Phi^{g}=\left(\begin{array}{ll}
\Phi_{11}^{-1} & 0 \\
0 & 0
\end{array}\right)
$$

where $\operatorname{rank}\left(\Phi_{11}\right)=\operatorname{rank}(\Phi)$.

These two approaches are theoretically equivalent, but small changes may occur in practice since $R(\hat{\Phi})$ is in general different from $R(\Phi)$. The reduced problem has some obvious computational savings, but a detailed intercomparison has yet to be made. For an extensive treatment of the MLM applied to wavenumber/frequency spectrum estimation, we refer to Capon (1979) and McDonough (1979).

Let $\Phi_{D}=\int_{0}^{2 \pi} h(\theta) h^{H}(\theta) D(\theta) d \theta$ and consider the operator $M: D \rightarrow D_{\text {MLM }}$ defined by

$$
D \rightarrow \Phi_{D} \rightarrow D_{\mathrm{MLM}}=\kappa / h^{H} \Phi_{D}^{g} h
$$

The operator is not generally $1-1$ since any function orthogonal to $h_{i} h_{j}^{*}$ for all $1 \leqslant i, j \leqslant N$ may be added to $D$ without affecting the result $M(D)$ as long as $D$ is positive and has integral equal to one. Moreover, $D_{\text {MLM }}$ is typically smeared compared to the true $D$. One obvious way of improving the estimate would be to solve the equation

$$
M(D)=\hat{D}_{\mathrm{MLM}}
$$

where $\hat{D}_{\text {MLM }}$ is the MLM estimate based on the data. This idea was basically introduced by Pawka (1983) and Oltman-Shay and Guza (1984) and is denoted the Iterative Maximum Likelihood Method (IMLM). Numerical experiments with the iterative scheme

$$
\begin{aligned}
D_{n+1} & =D_{n}+\omega_{R}\left(\hat{D}_{\mathrm{MLM}}-M\left(D_{n}\right)\right), \\
D_{0} & =\hat{D}_{\mathrm{MLM}}
\end{aligned}
$$

where $\omega_{R}$ is a relaxation parameter and the integral of $D_{n}$ is normalized to 1 after each iteration are shown in $\S 4$.

\subsection{Maximum entropy methods}

The concept of maximum entropy spectral estimation (MEM) has become popular in many applications. For one-dimensional time series the procedure is equivalent to fitting an autoregressive (AR) model to the data, commonly referred to as the Box-Jenkins approach. Unfortunately, for general arrays, no manageable algorithm for an MEM estimate seems to have been worked out (McDonough, 1979 , p. 233).

In our setting, the MEM estimate for the directional distribution is equivalent to

$$
-\int_{0}^{2 \pi} \log (D(\theta)) d \theta=\max
$$

while

$$
\int_{0}^{2 \pi} h(\theta) h^{H}(\theta) D(\theta) d \theta=\Phi
$$


The introduction of a Hermitian matrix of Lagrange multipliers, $\Lambda$, leads to the functional

$$
H(D, \Lambda)=-\int_{0}^{2 \pi} \log (D(\theta)) d \theta+\int_{0}^{2 \pi} h^{H}(\theta) \Lambda h(\theta) D(\theta) d \theta-\operatorname{tr}(\Lambda \Phi)
$$

Variation with respect to $D$ immediately yields

$$
D(\theta)=1 / h^{H}(\theta) \Lambda h(\theta)
$$

and the condition $D(\theta)>0$ is taken care of by requiring $\Lambda>0$. The constraints are equivalent to

$$
\int_{0}^{2 \pi} \log \left(h^{H}(\theta) \Lambda h(\theta)\right) d \theta-\operatorname{tr}(\Lambda \Phi)=\max
$$

No simple, general solution of (3.26) appears to be known. The MLM estimate is recovered if the integral in (3.26) is replaced by $-\log |\Lambda|$.

A discussion of the uniformly spaced line array which in principle is similar to one-dimensional AR-spectral estimation is given in McDonough (1979), § 6.4.4. The one-dimensional case is nevertheless interesting since 'single point triplets' of wave gauges produce estimates of the Fourier coefficients of $D$. Single point triplets include heave/pitch/roll buoys (Barstow and Krogstad, 1984) and vector measuring current meters. Small arrays are also often processed to give Fourier coefficients rather than the directional distribution itself (Borgman, 1979).

We recall that

$$
-\int_{0}^{2 \pi} \log D(\theta) d \theta=\max
$$

subject to

$$
\begin{gathered}
D(\theta)>0, \\
\int_{0}^{2 \pi} D(\theta) d \theta=1, \\
\int_{0}^{2 \pi} \exp (-\operatorname{in} \theta) D(\theta) d \theta=c_{n}, 1 \leqslant n \leqslant N,
\end{gathered}
$$

is

$$
D(\theta)=\frac{1}{2 \pi} \frac{\sigma_{e}^{2}}{\left|1-\phi_{1} \exp (-\mathrm{i} \theta)-\cdots-\phi_{N} \exp (-\mathrm{i} N \theta)\right|^{2}}
$$

where the parameters $\phi_{1} \cdots \phi_{N}$ and $\sigma_{e}^{2}$ are obtained from the Yule-Walker equations (Box and Jenkins, 1976):

$$
\begin{aligned}
& {\left[\begin{array}{lll}
1 & c_{1}^{*} & c_{N-1}^{*} \\
c_{1} & & c_{1}^{*} \\
c_{N-1} & c_{1} & 1
\end{array}\right]\left[\begin{array}{l}
\phi_{1} \\
\vdots \\
\phi_{N}
\end{array}\right]=\left[\begin{array}{l}
c_{1} \\
\vdots \\
c_{N}
\end{array}\right]} \\
& \sigma_{e}=1-\phi_{1} c_{1}^{*}-\cdots-\phi_{N} c_{N}^{*}
\end{aligned}
$$

The estimates for the Fourier coefficients entering Eqn. (3.29) have to be positive definite. This requirement may easily be satisfied for single point triplets where the most common estimates of $c_{1}$ and $c_{2}$ have turned out to be ML estimates in the sense of Eqn. (3.5). See Lygre and Krogstad (1986) for further applications of the MEM to data from a heave/pitch/roll buoy. 


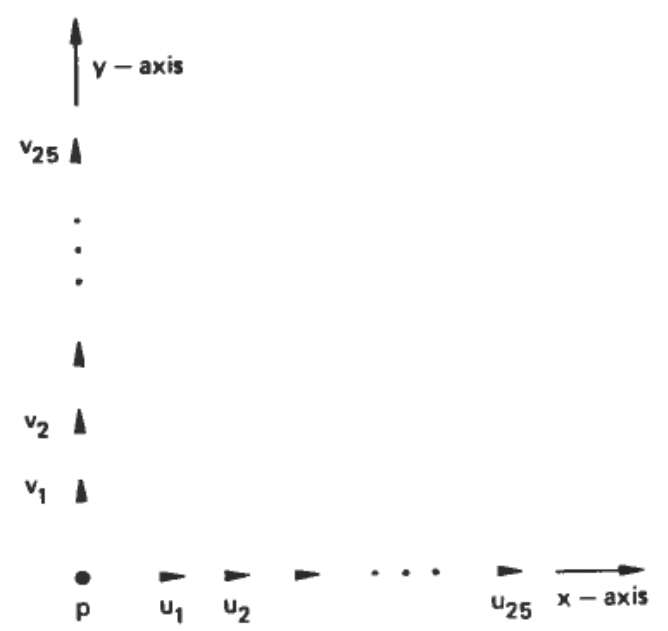

Figure 2. The CUMEX ADCM array configuration: a pressure cell $(p)$ at the origin and up to 25 velocity recordings along two orthogonal horizontal axes. The distance from the origin to the nearest velocity measurement is $6.9 \mathrm{~m}$ and the distance between the velocity measurements $4.6 \mathrm{~m}$. The instrument is situated on a platform leg, $14.2 \mathrm{~m}$ below mean water level.

\section{Applications}

The dimensionless parameter $\Delta$ determining the resolution of a spatial array of wave gauges is the characteristic array dimension times the wavenumber. For ocean waves the typical frequency range is $0.05-0.5 \mathrm{~Hz}$, and since $k=\omega^{2} / g$ in deep water, $\Delta$ will vary about two decades. One therefore has to expect a variation in resolution with frequency. A second matter to consider is varying directional sensitivity with the orientation of the array.

We shall now illustrate these aspects of the MLM and the IMLM algorithms for the ADCM 'array' from CUMEX displayed in Fig. 2. The ADCM measures the water velocity along a narrow beam of sound by observing the Doppler shift in the backscatter from impurities in the water masses. Time-gating allows recordings from different locations along the beam. In addition, a pressure cell is situated at the instrument. See Gordon and Skorstad (1985) and Krogstad, Miller and Gordon (1988) for further description of CUMEX.

The direction dependent part of cross-spectral matrices from the array has the structure

$$
\Phi=\left[\begin{array}{lll}
\Phi_{p p} & \Phi_{u p}^{H} & \Phi_{v p}^{H} \\
\Phi_{u p} & \Phi_{u u} & \Phi_{u v}^{H} \\
\Phi_{v p} & \Phi_{u v} & \Phi_{v v}
\end{array}\right]=\int_{0}^{2 \pi} h(\theta) h^{H}(\theta) D(\theta) d \theta
$$

where

$$
h(\theta)=\left[\begin{array}{c}
1 \\
\cos \theta \exp (\mathrm{i} \Delta(1) \cos \theta) \\
\cos \theta \exp (\mathrm{i} \Delta(2) \cos \theta) \\
\vdots \\
\cos \theta \exp (\mathrm{i} \Delta(M) \cos \theta) \\
\sin \theta \exp (\mathrm{i} \Delta(1) \sin \theta) \\
\vdots \\
\sin \theta \exp (\mathrm{i} \Delta(M) \sin \theta)
\end{array}\right]
$$



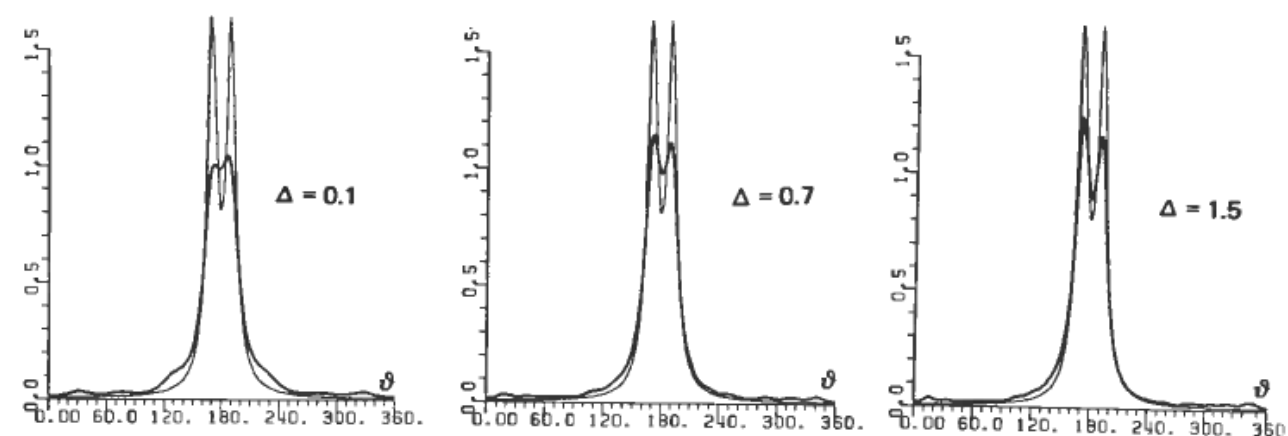

Figure 3. MLM estimate (thick line) of a narrow double peaked distribution (thin line) with a peak separation of $20^{\circ}$. The included channels are $p, u_{1}, \ldots, u_{10}, v_{1}, \ldots, v_{10}$ and $\Delta=k \cdot a$, cf. Figure 2 .

and $\Delta(j)=|k| a\left(j+\frac{1}{2}\right), a=4.6 \mathrm{~m}$, and $\Phi_{p p}$ is $1 \times 1, \Phi_{u p}$ and $\Phi_{v p} M \times 1$, and $\Phi_{u u}$, $\Phi_{v v}$ and $\Phi_{u v} M \times M, M=25$.

The full $\Phi$ matrix is close to being singular, and any attempts to numerically invert the matrix for the frequency range in question will fail. In order to become familiar with the array, a series of numerical simulations have been carried out using subsets of the available channels.

In general, the performance of the array improves as $\Delta$ increases, but if $\Delta$ becomes too large, the estimate deteriorates due to poorly correlated channels in the presence of noise. The varying resolution for the MLM estimate $D_{\mathrm{MLM}}=\kappa / h^{H} \Phi_{D}^{g} h$ corresponding to a narrow double-peaked distribution $D$ is shown in Fig. 3.

Although some instruments like the single point triplets are easily seen to be independent of orientation, varying directional sensitivity has to be expected in general. An example is shown in Fig. 4. The estimate varies to some extent with the direction of the incoming waves although the observed variation is roughly within the sampling variability if the degrees of freedom in the spectral estimates are of the order of 50-100. Numerical experiments indicate that the sampling variability for the IMLM estimates is comparable or slightly larger than indicated by Eqn. (3.11). These results will be published elsewhere.

Since we are using pivoting in the factorization of $\Phi$ (and $\hat{\Phi}$ ), a submatrix is actually used in the estimate. Iterations (Eqn. (3.22)) on the MLM estimate shown
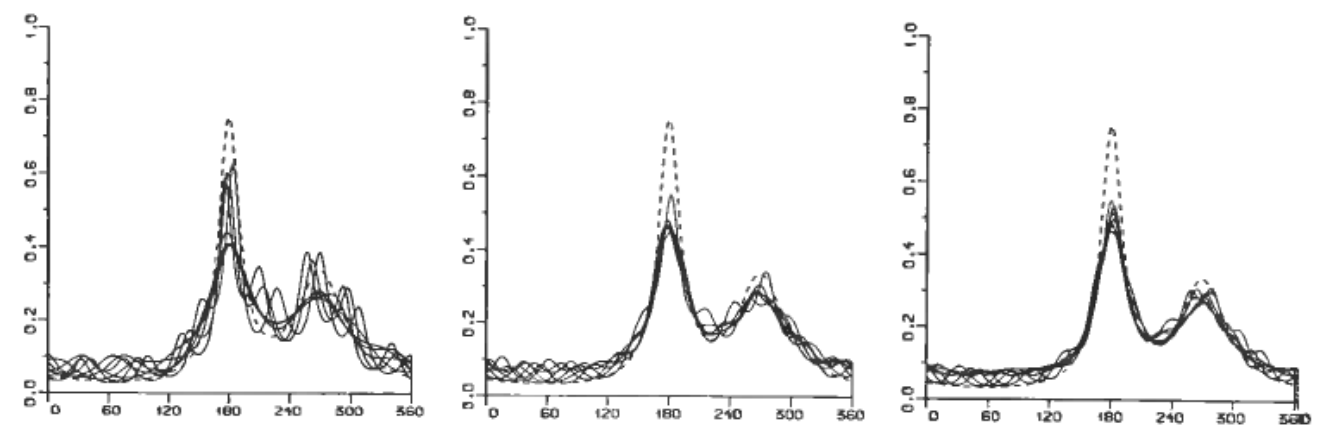

Figure 4. Effects of array orientation: MLM estimates for identical wave trains arriving at directions varying in steps of $50^{\circ}$. Including channels: $p, u_{1}, \ldots, u_{5}, v_{1}, \ldots, v_{5} . \Delta=0 \cdot 1$ (left), $\Delta=0.4$ (middle) and $\Delta=1.0$ (right). Input distribution shown with a dashed line. 

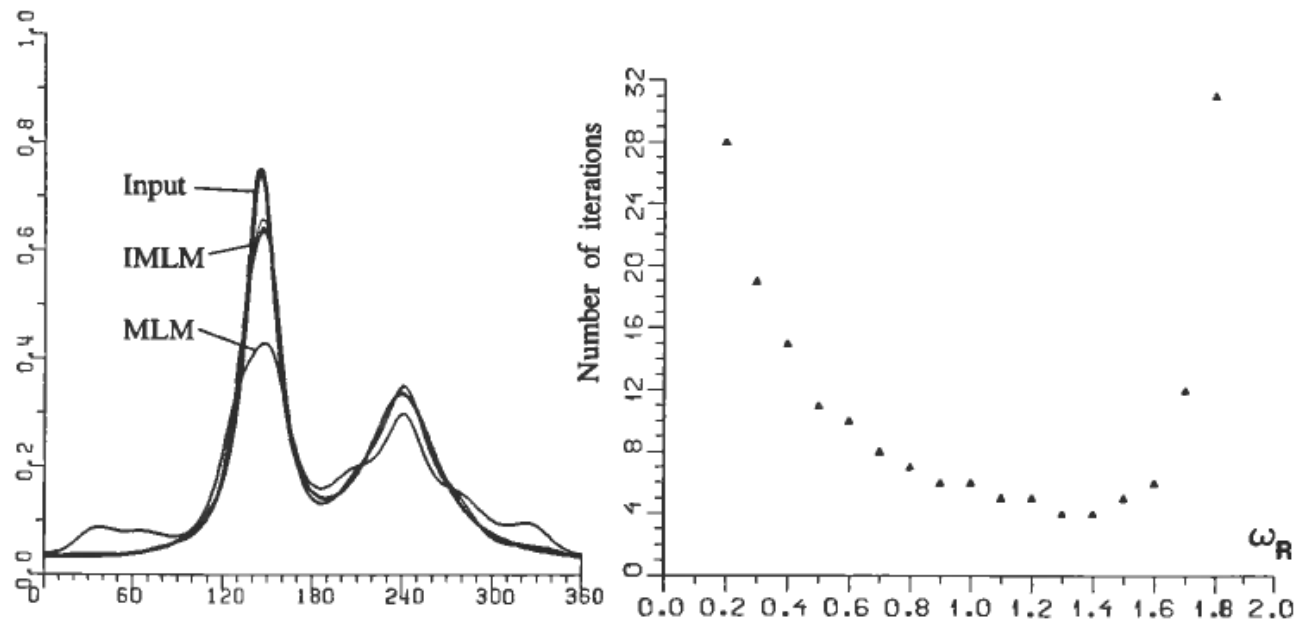

Figure 5. Left: Superimposed IMLM estimates for various values of $\omega_{R} . \Delta=0 \cdot 5$, input channels $p, u_{1}, \ldots, u_{3}, v_{1}, \ldots, v_{3}$, stopping criterion $\max |M(D)-D|<0 \cdot 01$. Number of necessary iterations as a function of $\omega_{R}$ shown to the right.

below are therefore based on the actual channels used in the MLM-estimate, and not the full set of channels. Experiments varying the relaxation parameter, $\omega_{R}$, showed that the iteration converged even for $\omega_{R}$ slightly greater than 1. However, as divergence occurred rather abruptly when $\omega_{R}$ was increased much beyond $1, \omega_{R}=1$ appears to be reasonable.

An example of IMLM estimates for synthetic data is shown in Fig. 5. It is clear that the IMLM estimate in this case is superior to the MLM estimate. Although extensive numerical experiments using a slightly different iteration scheme were reported by Pawka (1983), no rigorous analysis of the convergence performance of the IMLM has been carried out.

The CUMEX measurement site at the Odin field in the Vikingbanken area is shown in Fig. 6. We note the narrow opening between the Shetland and Orkney islands towards the Atlantic. Figure 7 shows an example of time series observed with the ADCM. The time axis is horizontal with the pressure channel scaled and shown in the centre. The velocity channels along the $x$-axis are shown in increasing distance from the origin downwards and similarly for the $y$-axis channels upwards. The outermost channels suffer from occasional data errors due to low signal to noise ratio. Waves, and in particular groups of waves travelling through the array are clearly seen. Moreover, a high amount of correlation from channel to channel is evident. The correlation in the significant parts of the spectra is also obvious, see Fig. 8. The flat noise level seen in the spectra indicates an RMS random error of about $0.05 \mathrm{~m} / \mathrm{s}$. This limits the high frequency response of the system to about $0.2 \mathrm{~Hz}$, that is, ocean waves longer than approximately $35 \mathrm{~m}$.

Figure 9 shows contour plots of the directional spectrum obtained by the MLM and IMLM after two and four iterations. We note that when going from zero to two iterations, the estimate appears to improve significantly, whereas only minor changes are seen when going from two to four iterations. A typical error trace is shown in Fig. 10. After an initial phase with geometric decay, the error stabilizes. This suggests running 3-5 iterations on the MLM estimate in order to improve the 


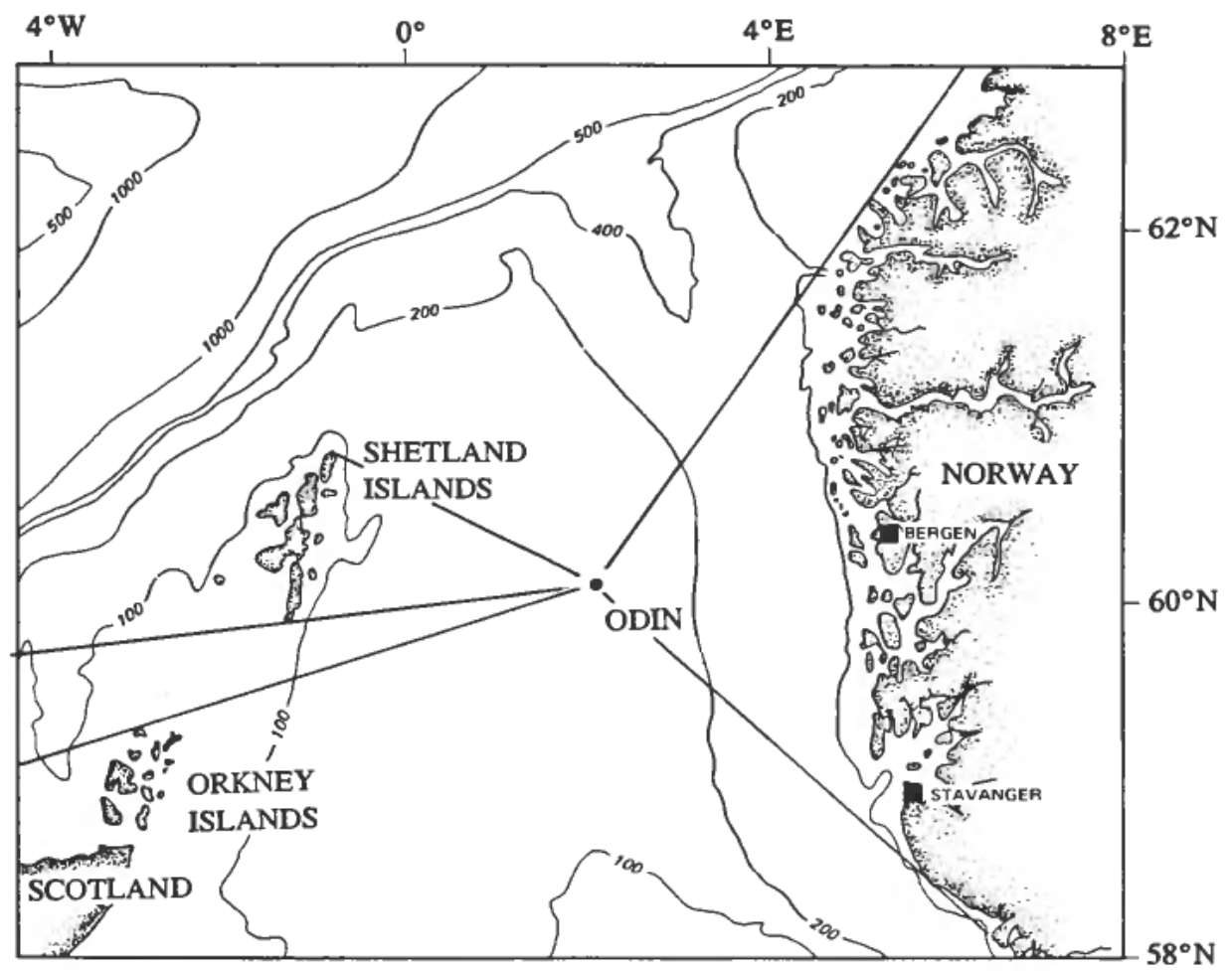

Figure 6. The CUMEX measuring site at the Odin field in the Norwegian Sea. Note the narrow opening towards the Atlantic between the Shetland and Orkney Is.

estimate while not necessarily reaching an overall fix-point. Note that Atlantic swell is visible in the spectrum at $0 \cdot 07-0 \cdot 1 \mathrm{~Hz}$ at a direction of around $260^{\circ}$.

\section{Discussion}

The Iterative Maximum Likelihood Method (IMLM) offers an algorithm for extracting directional wave spectra which is completely general with respect to array geometry and the type of sensors. However, the large spatial oversampling of the CUMEX ADCM 'array' caused failures in the first attempts to apply the MLM. Near singular cross-spectral matrices lead to numerical problems, and it became evident that a procedure was needed for selecting a reasonable subset of channels. The Cholesky diagonal pivoting algorithm offers a way to select channels in an order which adapts to the actual data matrix.

As the cross-spectral matrices get gradually more singular when the frequency drops, it often turns out that different channels are used for different frequencies. For the practical applications of the method, it is conforting that 3-5 iterations on the MLM estimate appear to suffice. Pawka (1983) applied 50 iterations for a slightly different scheme, and this would prohibit the use of the method on large data sets. The method lends itself naturally to real time processing. Computation of crossspectra is routine and the iteration starting from the MLM estimate is only based on the array geometry.

Although the CUMEX system was designed for current measurements, the instrument configuration seems to be promising for directional wave measurements. 


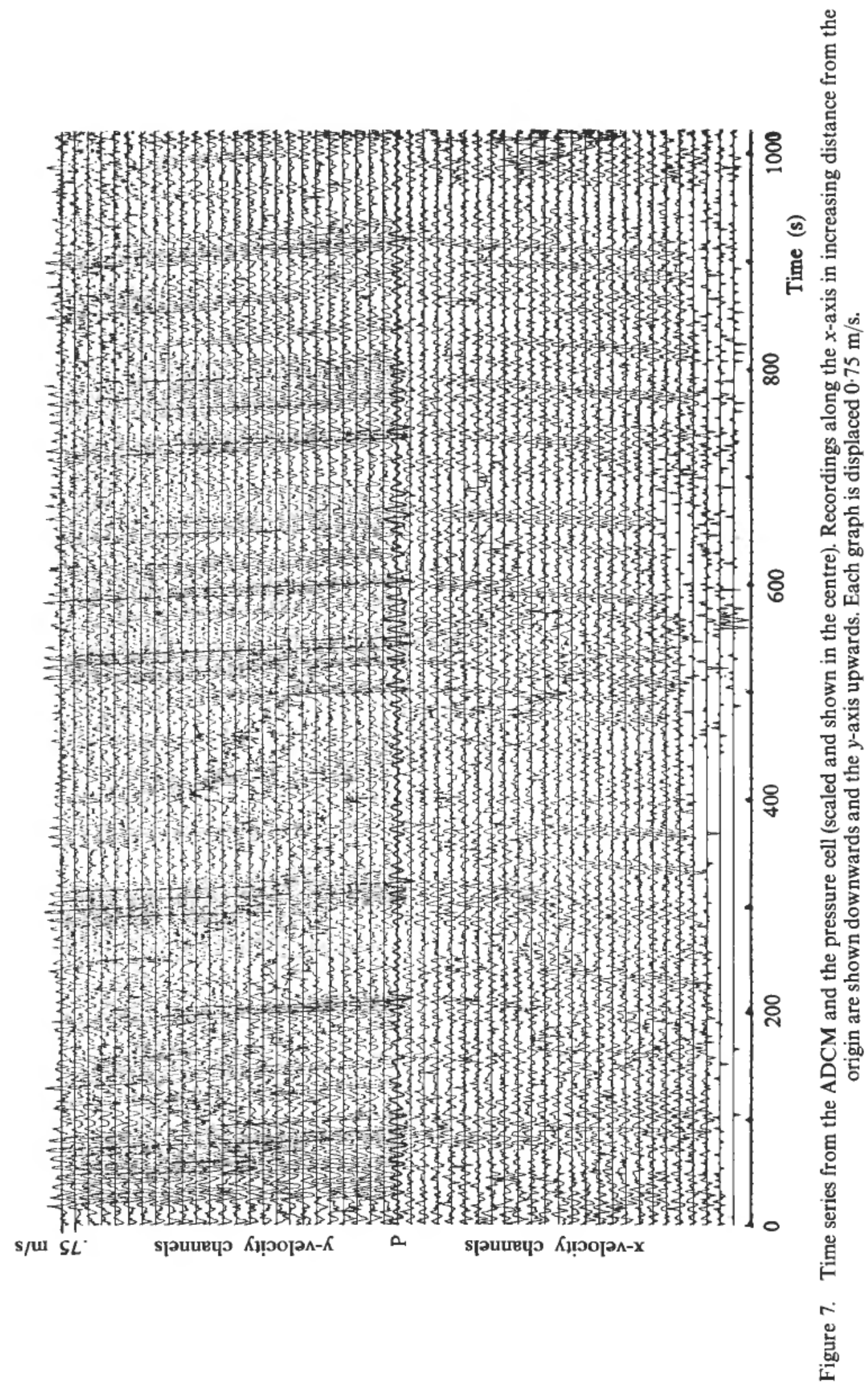




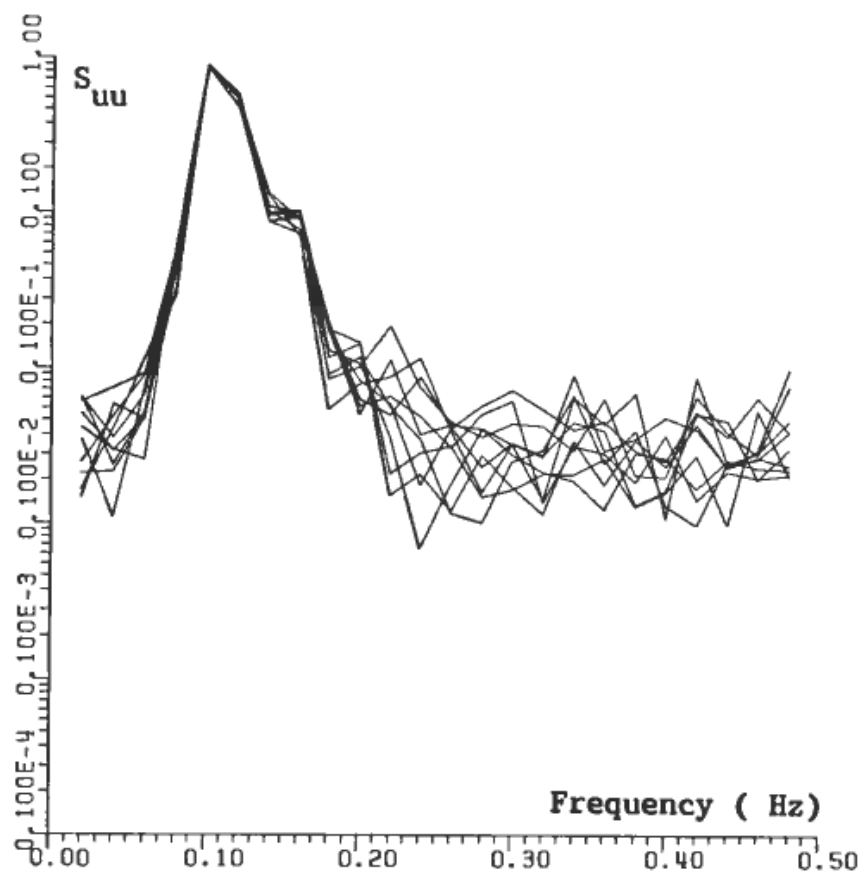

Figure 8. Example of velocity autospectra. The significant parts of the spectra are heavily correlated due to the correlation between the time series.

It has turned out that the pressure gauge at the instrument is superfluous (Krogstad et al. 1988), and if this is omitted, we may have a platform-based instrument which is measuring away from the influence of the platform. In a system specially designed for directional wave measurements, 4-6 measurement positions along each beam would be sufficient.
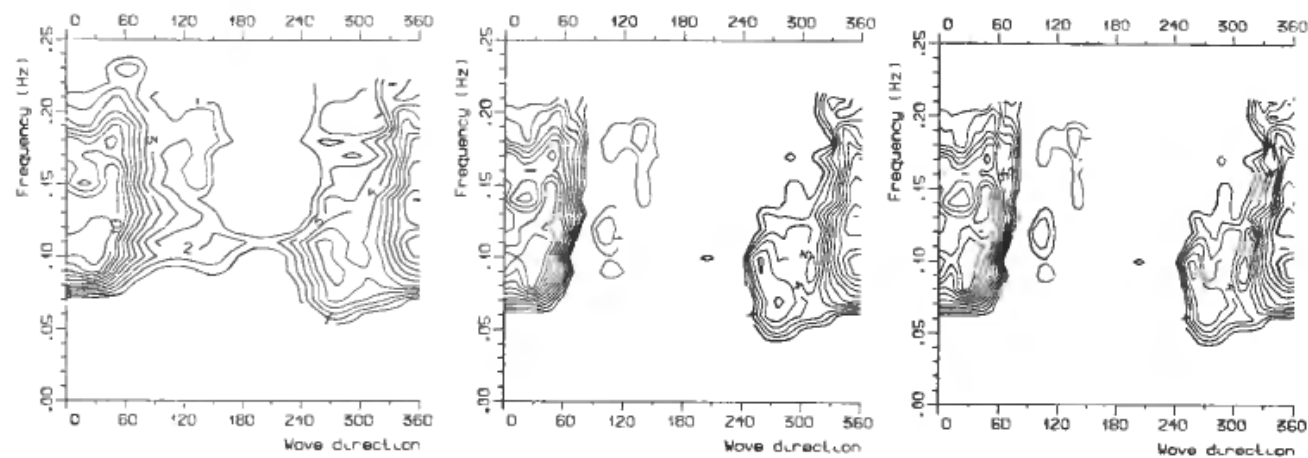

Figure 9. Contour plots of directional estimates based on real data using channels $p, u_{1}, u_{4}$, $u_{7}, u_{10}, v_{1}, v_{4}, v_{7}$ and $v_{10}$. Left: MLM, middle: MLM +2 iterations, right: MLM + 4 iterations. 


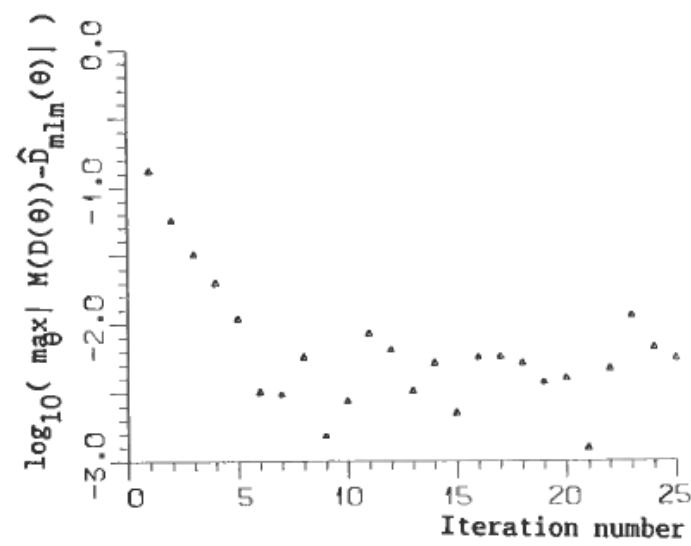

Figure 10. Characteristic behaviour of the error, $\max \left|M(D)-D_{\mathrm{MLM}}\right|$, in the IMLM traced from iteration to iteration for real data.

\section{Acknowledgement}

This study is in part supported by Esso Norge a.s.

\section{REFERENCES}

Barstow, S. F., and Krogstad, H. E. (1984), General analysis of directional ocean wave data from heave/pitch/roll buoys, Modeling, Identification and Control, 5, 47-70.

Borgman, L. E. (1979), Directional spectra from wave sensors, in Ocean wave climate, Ed.: M. D. Earle and A. Malahoff. vol. 88, pp. 269-300 Marine Science Series, (Plenum Press, New York).

BORGMAN, L. E. (1982), Maximum-entropy and data-adaptive procedures in the investigation of ocean waves, Manuscript presented at the Second Workshop on Maximum Entropy and Bayesian Methods in Applied Statistics, Laramie, Wyoming.

Box, G. E. P., and Jenkins, G. M. (1976). Time Series Analysis, Forecasting and Control (Holden-Day Inc., San Francisco)

Brillinger, D. R. (1975). Time Series, Data Analysis and Theory (Holt Rinehart and Winston, Inc., New York).

CAPON, J. (1969). High resolution frequency-wavenumber spectrum analysis, Proc. IEEE, 57, $1408-1418$.

CAPON, J. (1979). Maximum-likelihood spectral estimation, in Nonlinear Methods in Spectral Analysis, pp. 155-179, edited by S. Heykin (Springer, Berlin, Heidelberg, New York).

DAVIES, R. E. and REGIER, L. A. (1977). Methods for estimating directional wave spectra from multi-element arrays, J. Mar. Res., 35, 453-477.

Gihman, I. I. and Skorohod, A. V. (1974). The Theory of Stochastic Processes, vol. I (Springer, Berlin, Heidelberg).

Gordon, R. L. and SKoRSTAD, H. E. (1985). North Sea current data will upgrade platform design, Ocean Industry, July 1985.

Hannan, E. J. (1970). Multiple Time Series (Wiley, New York).

ISOBE, M., KONDO, K., and HoRIKAWA, K. (1984). Extension of MLM for estimating directional wave spectra, Proc. Symp on Description and Modelling of Directional Seas, June 18-20, 1984, Lyngby, Denmark.

Jeffreys, E. R., Wareham, G. T., Ramsden, N. A., and Platts, M. J. (1981). Measuring directional spectra with the MLM, Proc. Directional Wave Spectra Applications, pp. 203-218, Berkeley, California, Publ. by ASCE, New York.

KINSMAN, B. (1965). Wind waves, (Prentice Hall, Inc., Englewood Cliffs, New Jersey). 
Krogstad, H. E., Miller, M. and Gordon, R. L. (1988). High resolution directional wave spectra from horizontally mounted acoustic Doppler current meters, to appear in $J$. of Atmospheric and Oceanic Techn., February, 1988.

LyGre, A. and Krogstad, H. E. (1986). Maximum entropy estimation of the directional distribution in ocean wave spectra, J. Phys. Ocean., 16, 2052-2060.

Mardia, K. V., Kent, J. T., and BibBy, J. M., (1979). Multivariate Analysis, (Academic Press, London).

MCDonough, R. N. (1979). Application of the maximum likelihood method and the maximum entropy method to array processing, in Nonlinear Methods in Spectral Analysis, pp. 181-244, edited by S. Haykin, (Springer, Berlin, Heidelberg, New York).

Oltman-Shay, J. and GuZA, R. T. (1984). A data-adaptive ocean wave directional-spectrum estimator for pitch/roll type measurements, J. Phys. Ocean., 14, 1800-1810.

PawKA, S. S. (1983). Island shadows in wave directional spectra, J. Geophys. Res. 88, 25792591.

PhIllips, O. M., 1977: The Dynamics of the Upper Ocean, 2nd Ed. (Cambridge University Press, Cambridge.) 